\title{
Elevation of sE-Selectin Levels from 2-24 Months Following Gestational Diabetes is Associated with Early Cardiometabolic Risk in Non-Diabetic Women
}

\author{
Sokup Alina1,2*, Ruszkowska Barbara ${ }^{3}$, Góralczyk Krzysztof ${ }^{3}$, Góralczyk Barbara ${ }^{3}$, Grabiec Marek ${ }^{4}$ and Rość Danuta $^{3}$ \\ ${ }^{1}$ Department of Gastroenterology, Angiology and Internal Diseases, Nicolaus Copernicus University, Dr J. Biziel University Hospital, Bydgoszcz, Poland \\ ${ }^{2}$ Department of Endocrinology, Dr J. Biziel University Hospital, Bydgoszcz, Poland \\ ${ }^{3}$ Department of Pathophysiology, Nicolaus Copernicus University, Dr A. Jurasz University Hospital, Bydgoszcz, Poland \\ ${ }^{4}$ Department of Obstetrics and Gynecology, Nicolaus Copernicus University, Dr J.Biziel University Hospital, Bydgoszcz, Poland
}

\begin{abstract}
Women with previous gestational diabetes (pGDM) represent a high risk group for type 2 diabetes and for cardiovascular disease in the future. The aim of current study was to assess selected parameters of dysfunction of endothelium such as concentration of soluble E-selectin (sE-selectin), soluble vascular cell adhesion molecule-1 (sVCAM-1), soluable intracellular adhesion molecule-1 (sICAM-1) in women with pGDM.

Subjects/Methods: Anthropometric parameters, glucose regulation, insulin resistance, lipid levels, inflammation and soluble plasma endothelial dysfunction. Biomarkers levels were evaluated in 85 women with pGDM and in the control group of 40 patients 2-24 months postpartum.

Results: The pGDM group consisted of $67 \%$ women with normal glucose regulation (pGDM-N) and $33 \%$ with pre-diabetic state (pGDM-P). The body mass index, waist circumference, fasting and 2 -h glucose during oral glucose tolerance test, sVCAM-1, sICAM-1, sE-selectin, tissue plasminogen activator antigen (tPA:Ag), high sensitivity C-reactive protein (hsCRP), total-, LDL-cholesterol and triglycerides/HDL-cholesterol ratio were higher in pGDM women compared with the controls. The pGDM-P group was characterized by higher triglycerides levels compared with the pGDM-N. In the pGDM group only the plasma level of sE-selectin was associated with high glucose concentration within the pre-diabetic range. SE-selectin level correlated with triglycerides, triglycerides /HDL-cholesterol ratio, plasminogen activator inhibitor-1 antigen (PAI-1:Ag) and SICAM-1. In stepwise models of logistic regression analysis, sE-selectin was associated with high levels of glucose independently of age, BMI, PAl-1: Ag and of hsCRP. This association was eliminated after adjustment for triglyceride level.
\end{abstract}

Conclusions: In non-diabetic women with pGDM higher plasma sE-selectin level is not independently associated with the higher risk of pre- diabetic state. We suggest that in the case of these women the higher plasma sE-selectin level is rather the marker of early cardiometabolic risk due to its association with parameters of insulin resistance.

Keywords: Gestational diabetes; Pre-diabetic state; Soluble E-selectin; Cardiovascular risk

\section{Introduction}

Women with the history of gestational diabetes mellitus (GDM) have a significantly increased risk of type 2 diabetes and of cardiovascular disease during the next years after delivery [1]. A very important problem is GDM and its future consequences since it is dramatically increasing global public health problem which requires specific preventive strategies.

In the years postpartum, women with the history of GDM have an increased cardiometabolic derangement, including vascular risk factors and early vascular dysfunction [2-4]. Several studies, which were performed early after delivery ( 6 weeks- 2 years), have found the association of cardiovascular risk factors with glucose intolerance and in particular with type 2 diabetes [5,6].

Retnakaran et al have observed that there is an increased prevalence of metabolic syndrome in the third months postpartum among women with even mild glucose intolerance during pregnancy. Thus, it is possible that different stages of glucose dysregulation diagnosed during pregnancy are associated with early cardiovascular risk postpartum [7]. It has been suggested that the association between pre-diabetic state or diabetes and cardiovascular disease may be mediated through endothelial dysfunction $[8,9]$.
Soluble forms of adhesion molecules are released from shedding or proteolytic cleavage from the endothelial cell surface and may reflect overexpression of their respective membrane-bound forms [10]. Circulating levels of some endothelial-derived factors, soluble cell adhesion molecules, von Willebrand factor (vWF), tissue plasminogen activator (t-PA) and plasminogen activator inhibitor type1 (PAI-1) have been linked to the risk of type 2 diabetes in populations without previous history of GDM $[8,11-13]$. There is few controversial data concerning the associations between levels of soluble adhesion molecules and glucose regulation status in women with positive history of GDM. Studies have simultaneously assessed the associations with soluble adhesion molecules and hemostatic endothelial activation

${ }^{*}$ Corresponding author: Alina Sokup MD, PhD, St. Ujejskiego 75, 86-141 Bydgoszcz, Poland, Tel: 0048509217 662; Fax: +48 5237149 12; E-mail: alinasokup@o2.pl

Received July 26, 2011; Accepted August 19, 2011; Published September 08, 2011

Citation: Alina S, Barbara R, Krzysztof G, Barbara G, Marek G, et al. (2011) Elevation of sE-Selectin Levels from 2-24 Months Following Gestational Diabetes is Associated with Early Cardiometabolic Risk in Non-Diabetic Women. J Diabetes Metab 2:138. doi:10.4172/2155-6156.1000138

Copyright: (c) 2011 Alina S, et al. This is an open-access article distributed unde the terms of the Creative Commons Attribution License, which permits unrestricted use, distribution, and reproduction in any medium, provided the original author and source are credited. 
biomarkers with pre-diabetic and proatherogenic state in women with the previous history of GDM [14-16].

In the current study we hypothesized that early (2-24 months) endothelial dysfunction is associated with the development of early glucose dysregulation and/or with other atherosclerosis risk factors. It may also be the potential target included in preventive strategies in women with the previous history of GDM. The aim of current study was to assess selected parameters of dysfunction of endothelium such as concentration of soluble E-selectin (sE-selectin), soluble vascular cell adhesion molecule-1 (sVCAM-1), soluble intracellular adhesion molecule-1 (sICAM) in women with previous gestational diabetes (pGDM) 2-24 months postpartum.

\section{Material and Methods}

\section{Patients and subjects}

The research was conducted at the Diabetes and Pregnancy Unit at the Dr J Biziel University Hospital in Bydgoszcz, Poland, between 2005 and 2007.

The control group consisted of 85 Caucasian women with single gestations complicated by previous GDM (pGDM) and of 40 women with normal glucose status during pregnancy (previous normal glucose tolerance -pNGT). The group was evaluated in the period of 2-24 months after delivery.

All subjects ingested at least $150 \mathrm{~g}$ carbohydrates a day and refrained from exercise for at least 3 days before the study. None of the participants had renal, hepatic or cardiovascular disease. None of them were taking medications that would have affected lipid or carbohydrate metabolism. Women with a family history of diabetes mellitus, with infectious processes, under stress as well as those who were smoking, were not included in the study.

GDM was diagnosed according to the modified criteria of the World Health Organization [17] after performing a fasting and 2-h $75 \mathrm{~g}$ oral glucose tolerance test (OGTT). These modified criteria, which are commonly used in Poland, are based on taking fasting glucose concentration of $<5.6 \mathrm{mmol} / \mathrm{l}$ as a norm. This test was carried out in all pregnant women with a screening test (GCT-50-g oral glucose challenge) showing 2-h glucose value equal or above $7.8 \mathrm{mmol} / \mathrm{l}$. The screening test was performed during the first prenatal visit in all study groups and was repeated in the $24-28$ weeks of gestation.

At the postpartum assessment all participants underwent a physical examination that included anthropometric measurements. Waist circumference was measured at the smallest circumference between the ribs and iliac crest. BMI was calculated as weight in kilograms divided by the squared height in meters.

The standard 75-g 2-h OGTT was performed in order to assess glucose tolerance after delivery. The current status of glucose regulation was defined according to the modified WHO recommendations.

Venous blood $(4.5 \mathrm{ml})$ for endothelial markers tests was collected in a fasting state into cooled tubes (Becton Dickinson Vacutainer ${ }^{\circ}$ System, Plymouth, UK) containing $0.13 \mathrm{~mol} / \mathrm{L}$ trisodium citrate (the final blood - anticoagulant ratio was 9:1) after $30 \mathrm{~min}$ of rest between 7.00 and $9.00 \mathrm{am}$ and after a $12 \mathrm{~h}$ overnight fast. The blood samples were immediately mixed and centrifuged at $3000 \mathrm{xg}$ at $+4^{\circ} \mathrm{C}$ for $20 \mathrm{~min}$. The obtained platelet-poor plasma was divided into 200 $\mu \mathrm{l}$ Eppendorf-type tubes and then samples were frozen at $-86^{\circ} \mathrm{C}$ until assayed, but no longer than six months. Blood for serum lipids and insulin concentration, high sensitivity C-reactive protein (hsCRP) was collected in a tube containing no anticoagulant (Becton Dickinson Vacutainer 17490 , Plymouth, UK) and the serum was separated by centrifuging at $2500 \mathrm{xg}$ for $15 \mathrm{~min}$ and kept at $+4^{\circ} \mathrm{C}$ until analysed.

Serum glucose levels in samples from the OGTT were analysed using the glucose hexokinase method on the automatic analyser (Olympus Diagnostica GmbH, Ireland). Serum insulin concentration was determined using an immunochemical assay (8K41 Architect Insulin, Abbott Diagnostics, Denka Seiken, Japan) performed in the Architect Insulin analyser (Abbott Diagnostics, Denka Seiken , Japan). Serum concentrations of triglycerides and total LDL and HDL cholesterol were measured by enzymatic techniques (Olympus Diagnostica $\mathrm{GmbH}$, Ireland) on the Olympus automatic analyser( Olympus Diagnostica GmbH, Ireland).

The concentration of t-PA:Ag was determined by Enzyme Linked Immunosorbent Assay (ELISA)- ASSERACHROM ${ }^{\circ}$ t-PA (Diagnostica Stago, Asnieres, France), PAI-1:Ag was determined by ELISA- ASSERACHROM PAI-1- (Diagnostica Stago, Asnieres, France), vWF:Ag was assessed by ELISA- STA-Liatest VWF:Ag (Diagnostica Stago, Asnieres, France), C-reactive protein (CRP) was measured with a high-sensitivity assay by test IMUCLONE ${ }^{\text {mit }}$ CRP (hs) ELISA, C-Reactive Protein - ADI, using reagents from the American Diagnostica. Soluble sICAM-1 and sVCAM-1 were measured by using reagents from Bender Medsystems (Biomedica, Poland), soluble E-selectin was determined by test: E-selectin (ELAM-1) Antibody using reagent from IBL (Hamburg, Germany).

The homeostasis model assessment of insulin resistance (HOMAIR) index was calculated as a whole-body insulin sensitivity index. The HOMA index was calculated by multiplying the fasting glucose level (milimoles per liter) by the fasting insulin level (microunits per mililiter) and dividing the product by 22.5 [18].

\section{Statistical methods}

The statistical analysis was carried out using Statistica 8,0 (StatStoft ${ }^{\oplus}$ Cracow, Poland). The analysed parameters were tested for normal distribution using the Shapiro-Wilk test. Variables were not normally distributed; U-Mann-Whitney test was used. Results shown are median (interquartile range) or frequencies (percentages). The p-values $<0.05$ were considered statistically significant. Spearman correlation coefficients were calculated to determine if there were associations between measured parametric values and sE-selectin level in the group with the history of GDM. To investigate the association between glucose level and plasma levels of biomarkers of endothelial dysfunction, all post-GDM women were divided into two subgroups in accordance with low and high glucose concentration within the pre-diabetic range. The high glucose subgroup comprised women with fasting glucose equal or above $6,4 \mathrm{mmol} / \mathrm{l}$ and $<7,8 \mathrm{mmol} / \mathrm{l}$ at 2 -hours after $75 \mathrm{~g}$ glucose challenge(OGTT), women with fasting glucose $<5,6 \mathrm{mmol} / \mathrm{l}$ and $>8,9 \mathrm{mmol} / \mathrm{l}$ at 2 -hours and women with fasting glucose equal or above $6,4 \mathrm{mmol} / 1$ and $>8,9 \mathrm{mmol} / \mathrm{l}$ at 2 -hours. Other post-GDM women were included into the low glucose subgroups. We compared plasma levels of endothelial dysfunction biomarkers in these subgroups. The relation of sE-selectin concentration to glucose concentration was examined by multiple logistic regression analysis in order to consider the effect of potential determinants of endothelial dysfunction and diabetes. In separate logistic regression models, appropriate interaction terms were introduced to model interactions between sE-selectin tertile and age ( $>30$ y.o. vs $<30$ y.o.), BMI $=/>25 \mathrm{~kg} /$ $\mathrm{m} 2 \mathrm{vs}<25 \mathrm{~kg} / \mathrm{m} 2$ ), HOMA-IR (high tertile vs low tertile), triglycerides 
(high tertile vs low tertile), PAI-1:Ag levels (high tertile vs low tertile) and hsCRP levels (high tertile vs low tertile).

\section{Results}

At the postpartum assessment of 85 women with the previous history of gestational diabetes mellitus (pGDM) and of 40 women with normal glucose regulation during pregnancy (previous normal glucose tolerance -pNGT group), 7 women $(8,24 \%)$ in pGDM group had impaired fasting glucose with normal glucose tolerance at 2 -h OGTT (IFG/NGT), 16 (18,82\%) normal fasting glucose with impaired glucose tolerance at 2-h OGTT (NFG/IGT), 5 (5,88\%) impaired fasting glucose with impaired glucose tolerance at 2-h OGTT(IFG/IGT) and 57 women $(67,06 \%)$ had normal glucose regulation (NFG/NGT). In the pNGT group all women were classified as NFG/NGT. Baseline characteristics of subjects with the history of GDM and normal control subjects are shown in Table 1.

Women with the previous history of gestational diabetes mellitus (pGDM) had significantly higher levels of fasting glucose, 2-h (OGTT) glucose, HbA1c, sVICAM-1, sICAM-1, sE-selectin, tPA:Ag and both BMI and waist circumference compared to women with normal glucose regulation during pregnancy(pNGT). The pGDM group was also characterized by significantly higher lipid levels; total cholesterol, LDL-cholesterol, triglycerides and lower HDL-cholesterol. These two groups were similar in terms of age, insulin, vWF:Ag, PAI-1:Ag and HOMA-IR index value (Table 1).

Women with pGDM having pre-diabetic state (pGDM-P) showed a metabolic pattern similar to women with pGDM having normal glucose regulation (pGDM-N) with the exception of higher fasting blood glucose levels, 2-hour (OGTT) glucose, higher fasting triglycerides, triglycerides/HDL-cholesterol ratio. Women with pGDM-P did not differ significantly from pGDM-N women in terms of: age, BMI, waist circumference, sVCAM-1, sICAM-1, sE-selectin, tPA:Ag, vWF:Ag, PAI-1:Ag, hsCRP, total-cholesterol, LDL- and HDL. Two parameters, fasting insulin and HOMA-IR, were higher in women with pGDM-P but this difference was not statistically significant $(0,05$ and 0,06 respectively). Women with pGDM, regardless of their current glucose status, had higher BMI, larger waist circumference, higher 2-hour glucose in OGTT, higher values of hsCRP, sICAM-1, sEselectin, tPA:Ag, total-, LDL- and HDL-cholesterol compared with the controls (Table 2).

Only sE-selectin levels were significantly higher in high glucose within tne pre-diabetic state subgroup compared with the low glucose subgroup. There were no significant differences between the levels of other biomarkers of endothelial dysfunction in the subgroups of women subjected to comparison (Table 3).

In the whole pGDM group sE-selectin was modestly and positively correlated with fasting triglycerides $(\mathrm{r}=0,32)$, fasting triglycerides/cholesterol-HDL ratio( $\mathrm{r}=0,2910), \mathrm{PAI}-1 \mathrm{Ag}(\mathrm{r}=0,2647)$, tPA:Ag $(r=0,2622)$ and sICAM-1 $(r=0,2504)$. There were no significant correlations between $\mathrm{sE}$-selectin level and age, BMI, waist circumference, fasting insulin, glucose at 0 and 2-h OGTT, HbAlc, sVCAM-1, vWF:Ag, total- ,LDL- , HDL-cholesterol as well as HOMAIR.

After adjustment for age, the level of sE-selectin was positively and significantly associated with high glucose values. Further adjustment for HOMA-IR attenuated this association, whereas further adjustment for triglyceride eliminated this association. RRs of high glucose in the high tertile compared with low tertile were the following: 2,41 (95\% CI $1,09-5,32, \mathrm{p}=0,0306)$ for age- $3,40(1,21-9,58, \mathrm{p}=0,0214)$ for age and BMI- $2,08(0,97-4,45, \mathrm{p}=0,0506)$ for age and HOMA-IR- $1,85(0,82$ $4,15, \mathrm{p}=0,133)$ for age and triglycerides- $2,48(1,08-5,71, \mathrm{p}=0,0328)$ for age and hsCRP- $2,86(0,98-8,32, \mathrm{p}=0,0531)$ for age, BMI and

\begin{tabular}{|c|c|c|c|}
\hline Variables (units) & pGDM (n=85) & pNGT $(n=40)$ & p-values \\
\hline Age (years) & $29.00(26.00,35.00)$ & $27.00(25.00,35.50)$ & 0.2040 \\
\hline BMI $\left(\mathrm{kg} / \mathrm{m}^{2}\right)$ & $23.68(20.96,27.54)$ & $22.00(20.31,24.33)$ & $0.0098^{*}$ \\
\hline Waist (m) & $0,80(0,73-0,92)$ & $0,74(0,69-0,78)$ & $0.0007^{*}$ \\
\hline $\mathrm{HbA}_{1} \mathrm{C}(\%)$ & $5.50(5.40-5.70)$ & $5.40(5.30-5.40)$ & $0.0025^{*}$ \\
\hline Fasting plasma glucose (mmol/l) & $4,50(4,61-5,33)$ & $4,72(4,50-4,78)$ & $0.0060^{*}$ \\
\hline 2-h (OGTT) plasma glucose (mmol/l) & $6.16(5,55-7,66)$ & $4,84(4,77-5,44)$ & $<0.0001^{*}$ \\
\hline Insulin (pmol/l) & $54,87(41,67-80,56)$ & $63,20(54,87-71,53)$ & 0.1755 \\
\hline sVCAM-1 (ng/ml) & $1113.53(419.60-1616.70)$ & $672.05(455.70-1113.46)$ & $0.0312^{*}$ \\
\hline sICAM-1 (ng/ml) & $294.89(238.73-364.19)$ & $140.40(121.34-179.90)$ & $<0.0001^{*}$ \\
\hline sE-selectin (ng/ml) & $28.13(19.17-43.95)$ & $20.90(17.11-26.01)$ & $0.0201^{*}$ \\
\hline tPA:Ag (ng/ml) & $4.89(3.53-7.66)$ & $3.50(2.66-4.64)$ & $0.0005^{\star}$ \\
\hline vWF:Ag (\%) & 104.30(96.20-122.30) & 104.87 (74.96- 125.67) & 0.4405 \\
\hline PAl-1:Ag (ng/ml) & 57.09 (46.28-79.43) & 71.36 (50.54- 81.80) & 0.1371 \\
\hline hsCRP $(\mu \mathrm{g} / \mathrm{ml})$ & $1.22(0.66-2.76)$ & $0.41(0.22-0.79)$ & $<0.0001^{*}$ \\
\hline Total-cholesterol (mmol/l) & $5,04(4,55-5,51)$ & $4,56(4,27-5.09)$ & $0.0027^{*}$ \\
\hline HDL- cholesterol (mmol/l) & $1,53(1,28-1,73)$ & $1,73(1,56-1,82)$ & $0.0012^{*}$ \\
\hline LDL- cholesterol (mmol/l) & $3,10(2,79-3,54)$ & $2,57(2,30-3,05)$ & $0.0001^{*}$ \\
\hline Triglyceride $(\mathrm{mmol} / \mathrm{l})$ & $0,97(0,78-12,83)$ & $0,86(0,67-1,05)$ & $0.0263^{*}$ \\
\hline Triglyceride/ HDL cholesterol & $1,52(1,06-2,63)$ & $1,11(0,89-1,50)$ & $0,0024^{*}$ \\
\hline HOMA-IR (\%) & $1.67(1.22-2.80)$ & $1.89(1.58-2.37)$ & 0.3973 \\
\hline NFG/IGT n (\%) & $16(18.82)$ & - & - \\
\hline IFG/NGT n (\%) & $7(8.24)$ & - & - \\
\hline IFG/IGT n (\%) & $5(5.88)$ & - & - \\
\hline NFG/NGT n (\%) & $57(67.06)$ & $40(100)$ & - \\
\hline
\end{tabular}

Values are median (IQR) or $n(\%)$. P values were from U-Mann Whitney test. * $p<0,05$

Table 1: Characteristics of women with previous history of GDM (pGDM) and control participants with normal glucose regulation during pregnancy (pNGT-group). 


\begin{tabular}{|c|c|c|c|c|}
\hline Variable (units) & pGDM-P n=29 & pGDM-N n=56 & pNGT N=40 & p-values \\
\hline Age (years) & $31.50(27.50-35.50)$ & $30.00(26.00-35.00)$ & $27.00(25.00-35.50)$ & 0.1145 \\
\hline BMI $\left(k g / m^{2}\right)$ & $25.12(21.83-29.04)^{b}$ & $24.44(21.26-27.22)^{a}$ & $22.00(20.31-24.33)$ & 0.0022 \\
\hline Waist circumference $(\mathrm{cm})$ & $80.00(73.00-96.00) b$ & $82.00(73.00$ - 90.00)a & $74.50(69.00-78.00)$ & 0.0010 \\
\hline Fasting plasma glucose (mmol/l) & $5.16(4.77-5.88)^{b, c}$ & $4.77(4.44-5.13)$ & $4.72(4.50-4.78)$ & 0.0002 \\
\hline 2-h (OGTT) plasma glucose (mmol/l) & $8.27(8.05-8.77)^{b, c}$ & $5.88(5.44-6.66)^{a}$ & $4.84(4.77-5.44)$ & $<0.0001$ \\
\hline Insulin (pmol/I) & $57.44(42.36-95.84)^{\mathrm{cc}}$ & $54.17(41.67-69.45)$ & $63.20(54.87-71.53)$ & 0.0353 \\
\hline sVCAM-1 (ng/ml) & $960.30(353.73-1638.03)$ & $1127.63(466.68-1582.58)^{a}$ & $672.05(455.70-1113.46)$ & 0.0553 \\
\hline sICAM-1 (ng/ml) & $295.90(238.80-363.38)^{b}$ & $299.28(243.46-364.19)^{a}$ & $140.40(121.34-179.90)$ & $<0.0001$ \\
\hline sE-selectin (ng/ml) & $35.80(26.37-43.65)^{b}$ & $28.57(20.35-42.54)^{a}$ & $20.90(17.11-26.01)$ & 0.0022 \\
\hline tPA:Ag (ng/ml) & $6.01(4.09-7.51)^{b}$ & $4.68(3.25-7.24)^{a}$ & $3.50(2.66-4.64)$ & 0.0005 \\
\hline vWF:Ag (\%) & $107.79(96.20-131.59)$ & $104.30(95.36-117.22)$ & $104.87(74.96-125.67)$ & 0.6498 \\
\hline PAl-1:Ag (ng/ml) & $57.67(49.44-83.75)$ & $57.09(42.85-85.12)$ & $71.36(50.54-81.80)$ & 0.2178 \\
\hline hsCRP ( $\mu \mathrm{g} / \mathrm{ml})$ & $1.30(0.81-2.86)^{\mathrm{b}}$ & $1.32(0.66-2.76)^{\mathrm{a}}$ & $0.41(0.22-0.79)$ & $<0.0001$ \\
\hline Total-cholesterol (mmol/l) & $5.33(4.84-5.92)^{b}$ & $5.02(4.50-5.48)^{a}$ & $4.56(4.27-5.09)$ & 0.0022 \\
\hline HDL- cholesterol (mmol/l) & $1.45(1.29-1.73)^{b}$ & $1.55(1.27-1.73)^{\mathrm{a}}$ & $1.73(1.56-1.82)$ & 0.0048 \\
\hline LDL- cholesterol (mmol/l) & $3.26(2.97-4.06)^{b}$ & $3.03(2.77-3.54)^{a}$ & $2.57(2.30-3.05)$ & $<0.0001$ \\
\hline Triglyceride (mmol/l) & $1.21(1.02-2.22)^{b, c}$ & $79.00(63.00-116.00)$ & $74.50(58.50-91.00)$ & 0.0004 \\
\hline Triglyceride/ HDL cholesterol & $1.83(1.35-4.06)^{b, c}$ & $1.33(0.95-2.30)$ & $1.11(0.89-1.50)$ & 0.0003 \\
\hline HOMA-IR (mmol/l) & $2.32(1.27-3.49)^{\mathrm{ccc}}$ & $1.64(1.22-2.15)$ & $1.89(1.58-2.37)$ & 0.0499 \\
\hline
\end{tabular}

P-values for testing over the three groups (ANOVA): $a-p<0.05$ controls versus previous GDM with normal glucose regulation; $b-p<0.05$ controls versus previous GDM with pre-diabetic state; $c-p<0.05$ previous GDM with normal glucose status versus previous GDM with prediabetic state, $c c-p=0,05, c c c-p=0,06$

Table 2: Clinical and laboratory data for participants in studied groups: pGDM-P, pGDM-N and pNGT as a control group.

triglycerides- $1,66(0,73-3,79, \mathrm{p}=0,2206)$ for age, triglycerides and HOMA-IR- 3,67 (1,05-12,85, p=0,0472) for age, BMI, HOMA-IR and hsCRP- 2,75 (1,15-6,54) for age and PAI-1:Ag- 2,14 (0,90-5,12) for age, PAI-1:Ag- and HOMA-IR- 1,89 (0,78-4,56) for age, PAI-1:Ag and HOMA-IR (Table 4).

\section{Discussion}

Among 85 apparently healthy women with the previous history of gestational diabetes (pGDM), 33\% showed pre-diabetic state; $19 \%$ impaired glucose tolerance, $8 \%$ impaired fasting glucose and $6 \%$ impaired fasting glucose with impaired glucose tolerance after two hours of oral glucose tolerance test. These results are in accordance with previous reports [5].

Our study have shown that these young adult non-diabetic women with pGDM had higher concentrations of cardiovascular risk factors in the early (average 10 months) assessment after delivery in comparison with women with normal glucose regulation during pregnancy. The pGDM population was characterized by higher values of body fat indexes as well as by levels of endothelial dysfunction parameters (sICAM-1, sVCAM-1, sE-selectin, tPA:Ag) and by low-grade inflammation (hsCRP) which coexisted with dyslipidemic lipids profile and with similar insulin resistance in comparison to the control group.

Interestingly, women with pGDM and pre-diabetic state had similar concentrations of soluble adhesion molecules (sICAM-1, sE-selectin) and tPA:Ag compared with women with the history of GDM and normal glucose regulation, regardless of the presence of higher glucose concentration, higher fasting triglyceride level and slightly higher insulin concentration and insulin resistance index. It is well known and generally accepted that endothelial dysfunction is associated with insulin resistance and with its related metabolic abnormalities [19-21].

Our results are inconsistent with one previous report suggesting elevated sE- selectin levels during 12-26 months after delivery, only in post-GDM women with abnormal glucose regulation. However, they are in agreement with other two studies presenting higher sEselectin levels irrespectively of glucose regulation status [14-16] and of metabolic abnormalities related to insulin resistance [16]. Moreover, results of these studies suggest that elevated level of sE-selectin in postGDM women is independent of time since delivery $[14,16]$.

The current study is the first one in which we observed higher plasma levels of sICAM-1, sE-selectin and tPA:Ag levels with simultaneously normal levels of PAI-1:Ag and vWF:Ag in non-diabetic post-GDM women with both abnormal and normal glucose regulation.

Several studies have shown that circulating levels of ICAM-1, E-selectin [8,11], tPA:Ag [12,13], vWF:Ag and PAI-1:Ag [12] have been linked to the risk of type 2 diabetes in populations without the previous history of GDM.

In our study we have found that in the case of women with a history of GDM, high glucose concentrations within the pre-diabetic range were associated only with higher sE-selectin levels. This association was attenuated by adjustment for HOMA-IR and was eliminated by adjustment for fasting triglycerides levels. Additionally, sEselectin levels correlated positively with other markers of endothelial dysfunction, tPA:Ag, sICAM-1 and with well-known biochemical markers of insulin resistance such as fasting triglycerides, triglycerides/ HDL-cholesterol ratio and PAI-1:Ag level. Recently, triglycerides/ HDL cholesterol ratio has been suggested to be a predictor of insulin resistance and of the proportion of small and dense LDL particles, which is characteristic of atherogenic phenotype of the metabolic syndrome [22].

Thus, our findings suggest that endothelial dysfunction in the early assessment (average 10 months) after delivery in non-diabetic women with the history of GDM is characterized by higher plasma levels of circulating ICAM-1, sE-selectin and tPA:Ag independent of glucose regulation status, age, body fat indexes values, inflammation and PAI-1:Ag level. These observations are in agreement with the previous studies of Bo et al performed 6.5 years after delivery in women with previous GDM. In this study, they obtained higher levels of sEselectin, sICAM-1 and higher intima media thickness (IMT) values characterized post-GDM women, even those without any components of the metabolic syndrome except for glycaemia [16]. In all post-GDM 


\begin{tabular}{|l|l|l|l|}
\hline Variable (units) & Low glucose $(\mathbf{n = 5 8 )}$ & High glucose $(\mathbf{n = 2 7})$ & $\boldsymbol{p}$ values \\
\hline sVCAM (ng/ml) & $849.78(550.70-1285.55)$ & $669.18(419.60-13.90)$ & 0.3610 \\
\hline sICAM $(\mathbf{n g} / \mathbf{m l})$ & $222.44(152.66-317.08)$ & $247.79(212.59-338.68)$ & 0.1066 \\
\hline sE-selectin $\mathbf{( n g} / \mathbf{m l})$ & $25.90(19.03-40.17)$ & $37.75(27.93-46.15)$ & $0.0314^{*}$ \\
\hline vWF: $\mathbf{A g}(\%)$ & $101.72(91.76-119.04)$ & $107.10(86.68-136.34)$ & 0.3457 \\
\hline tPA:Ag (ng/ml) & $4.05(2.63-6.05)$ & $4.92(3.69-6.68)$ & 0.0838 \\
\hline PAl-1:Ag (ng/ml) & $61.85(47.42-85.18)$ & $55.47(44.93-83.75)$ & 0.7013 \\
\hline
\end{tabular}

Values are median (IQR), p values were from U-Mann-Whitney test. * $\mathrm{p}<0,05$

Table 3: Plasma concentrations of endothelium markers according to low and high glucose concentration within the pre-diabetic range.

\begin{tabular}{|l|l|l|}
\hline Variable & OR $(\mathbf{9 5} \% \mathbf{C I})^{\mathbf{a}}$ & $\boldsymbol{p}$ values \\
\hline Model 1: age-adjusted & $2.41(1.09-5.32)$ & $0.0306^{*}$ \\
\hline Model 1 + BMI & $3.40(1.21-9.58)$ & $0.0214^{*}$ \\
\hline Model 1 + HOMA-IR & $2.08(0.97-4.45)$ & 0.0506 \\
\hline Model 1 + triglycerides & $1.85(0.82-4.15)$ & 0.1339 \\
\hline Model 1 + hsCRP & $2.48(1.08-5.71)$ & $0.0328^{*}$ \\
\hline Model 1 + BMI + triglycerides & $2.86(0.98-8.32)$ & 0.0531 \\
\hline Model 1 + triglycerides + HOMA-IR & $1.66(0.73-3.79)$ & 0.2206 \\
\hline Model 1 + BMI + HOMA-IR + hs CRP & $3.67(1.05-12.85)$ & $0.0472^{*}$ \\
\hline Model 1 + PAI-1:Ag & $2,75(1,15-6,54)$ & $0,0230^{*}$ \\
\hline Model 1 + PAI-1:Ag + HOMA-IR & $2,14(0,90-5,12)$ & 0,0847 \\
\hline Model 1 + PAI-1:Ag + HOMA-IR + triglycerides & $1,89(0,78-4,56)$ & 0,1529 \\
\hline
\end{tabular}

${ }^{a}$ compared with low tertile of E-selectin

Table 4: Logistic regression models for the association between E-selectin and high glucose concentrations within the pre-diabetic range.

women, sE-selectin, sICAM-1, interleukin-6 and hsCRP values were significantly associated with IMT, thus supporting associations of these biomarkers with pathogenesis of early atherosclerosis [16].

Our present findings contrast with the previous study of Lawrence et al. These authors have detected clinical and biochemical markers of insulin resistance but have not shown any associations between sEselectin levels and these markers [15]. Additionally, higher levels of sEselectin in post-GDM women were restricted to women with abnormal glucose regulation. Thus, discrepant results of current study and the study of Lawrence et al may suggest pathophysiological differences in populations subject to examinations [15].

The correlation between glucose and plasma levels of sE-selectin are consistent to some extent with the previous studies conducted on patients with type 2 diabetes [21,23]. Increased sE-selectin concentrations in these studies were related to hyperglycemia, hyperinsulinaemia and insulin resistance, whereas increased sICAM-1 plasma concentrations mainly to hyperglycaemia [21]. Thus, it is possible that a specific relation between early atherogenic dyslipidemia, plasma sE-selectin levels and glucose concentration may be the characteristic feature of proatherogenic state in non-diabetic postGDM women which is partially similar to those in type 2 diabetes [21,23].

Another interesting finding in this study is the relation between high glucose levels within the pre-diabetic range and higher levels of sE-selectin which is dependent on fasting triglycerides and partially basal insulin resistance (HOMA-IR). It is suggested that non-diabetic post-GDM women, both overweight/obese and of normal weight show at least some factors of insulin resistance [19] regardless of glucose regulation status $[15,16]$. The severity of insulin resistance in women with GDM is also associated with the future risk of type 2 diabetes after delivery [24]. Thus, this latter mechanism and /or its related metabolic abnormalities may be a link between gestational hyperglycemia and the risk of diabetes post delivery. Interestingly, our results suggest a more important pathophysiological role for triglycerides than the insulin resistance in relation between sE-selectin levels and glucose.

There are possible explanations of the observed relation between high glucose within the pre-diabetic range and the higher plasma levels of sE-selectin. Firstly, slightly increased insulin resistance, disproportionally elevated plasma triglycerides and non-esterified fatty acids are associated with vascular endothelial dysfunction and with higher glucose due to insulin resistance and lipotoxic effects. Elevated triglyceride levels, decreased HDL cholesterol and higher levels of oxidized fatty acids may increase the expression of cell adhesion molecules [20]. Secondly, endothelial dysfunction is the primary mechanism and the cause of insulin resistance; elevation of triglycerides and non-esterified fatty acids aggravate insulin resistance and lead to hyperglycemia [25]. Finally, slightly elevated fasting triglyceride levels are associated with increased hepatic fat content [26], insulin resistance, dysfunction of endothelium and hyperglycemia. In this last metabolic situation, higher plasma levels of sE-selectin are secondary to higher triglycerides and/or insulin resistance. The impaired vascular endothelial function and increased arterial stiffness during and post pregnancies complicated by GDM were observed by several previous researchers $[2,3,27,28]$. Latest studies show the association between higher triglyceride levels at GDM diagnosis both with its prevalence [29] and with early atherosclerosis [4]. Interestingly, results of the study of Wiznitzer et al revealed that higher triglyceride levels were associated with the prevalence of GDM or preeclampsia [29]. Thus, it is possible that the relation between fasting triglycerides, sE-selectin and glucose may exist prior to or appears during pregnancy and persists post delivery. Increased metabolic demands of pregnancy may reveal this mechanism and lead to the development of both gestational hyperglycemia and early vascular damage during pregnancy as well as after delivery [30].

In conclusion, soluble adhesion molecules and tPA:Ag a hemostatic parameter of endothelial dysfunction, were raised in non-diabetic women with previous GDM irrespective of glucose regulation status in the assessment performed 2-24 months post delivery. Only sEselectin was associated with higher risk of pre-diabetic state. Results of our study suggest that in post-GDM women this latter association is independent of age, body mass index, inflammation and PAI-1:Ag levels but is strictly dependent on higher triglyceride levels and to a minor extent on higher insulin resistance. Thus, these findings do not support the role of sE-selectin as an independent marker of early abnormalities of glucose regulation or the future risk of diabetes in this group of women. Results of current data suggest the role of sE-selectin as an early marker of cardiometabolic risk because of its association with markers of insulin resistance. Interventions that improve metabolism of triglycerides and/or insulin sensitivity in the case of post-GDM women early after delivery could have beneficial effects on the progression of early phase of development of cardiometabolic syndrome.

\section{References}

1. Shah BR, Retnakaran R, Booth GL (2008) Increased risk of cardiovascular disease in young women following gestational diabetes. Diabetes Care 31 : 1668-1669.

2. Heitritter SM, Solomon CG, Mitchell GF, Skali-Clunis N, Seely EW (2005) Subclinical inflammation and vascular dysfunction in women with previous gestational diabetes mellitus. J Clin Endocrinol Metab 90: 3983-3988.

3. Anastasiou E, Lekakis J, Alevizaki M, Papamichael CM, Megas J, et al. (1998) Impaired endothelium-dependent vasodilatation in women with previous gestational diabetes. Diabetes Care 21: 2111-2113. 
Citation: Alina S, Barbara R, Krzysztof G, Barbara G, Marek G, et al. (2011) Elevation of sE-Selectin Levels from 2-24 Months Following Gestational Diabetes is Associated with Early Cardiometabolic Risk in Non-Diabetic Women. J Diabetes Metab 2:138. doi:10.4172/2155-6156.1000138

Page 6 of 6

4. Tarim E, Yigit F, Kilicda GE, Bagis F, Damircan S, et al. (2006) Early onset of subclinical atherosclerosis in women with gestational diabetes mellitus. Ultrasound Obstet Gynaecol 27: 177-182.

5. Morimitsu IK, Fusaro AS, Sanchez VH, Hagemann CC, Bertini AM, et al. (2007) Fibrynolytic dysfunction is associated to components of insulin resistance and early type 2 diabetes in Latino women with previous gestational diabetes. Diabetes Res Clin Pract 78: 340-348.

6. Pallardo E, Herranz L, Garcia-Ingelmo T, Grande C, Martin-Vaquero P, et al. (1999) Early postpartum metabolic assessment in women with prior gestational diabetes. Diabetes Care 21: 1053-1058.

7. Retnakaran R, Shah BR (2009) Mild glucose intolerance in pregnancy and risk of cardiovascular disease: a population-based cohort study. CMAJ 181: 6-7.

8. Meigs JB, Hu FB, Rifai N, Manson JE (2004) Biomarkers of endothelial dysfunction and risk of type 2 diabetes mellitus. JAMA 291: 1978-1986.

9. Suzuki T, Hirata K, Elkind MS, Jin Z, Rundek T, et al. (2008) Metabolic syndrome, endothelial dysfunction, and risk of cardiovascular events; The Northern Manhattan Study. Am Heart j 156: 405-410.

10. Price DT, Loscalzo J (1999) Cellular adhesion molecules and atherogenesis. Am J Med 107: 85-97.

11. Song Y, Manson JE, Tinker L, Rifai N, Cook NR, et al. (2007) Circulating levels of endothelial adhesion molecules and risk of diabetes in an ethnically diverse cohort of women. Diabetes 56: 1898-1904.

12. Meigs JB, O'Donnel CJ, Tofler GH, Benjamin EJ, Fox CS, et al. (2006) Hemostatic markers of endothelial dysfunction and risk of incident type 2 diabetes: The Framingham Offspring Study. Diabetes 55: 530-537.

13. Eliasson MC, Jansson JH, Lindahl B, Stegmayr B (2003) High levels of tissue plasminogen activator (t-PA) antigen precede the development of type 2 diabetes in a longitudinal population study. The Northern Sweden MONICA study. Cardiovasc Diabetol 2: 19-25.

14. Kautzky-Willer A, Fasching P, Jilma B, Waldhausl W, Wegner OF (1997) Persistent elevation and metabolic dependence of circulating E-selectin after delivery in women with gestational mellitus. J Clin Endocrinol Metab 82: 4117 4121.

15. Lawrence NJ, Kousta E, Penny A, Millauer B, Robinson S, et al. (2002) Elevation of soluble E-selectin levels following gestational diabetes is restricted to women with persistent abnormalities of glucose regulation. Clin Endocrinol 56: $335-340$

16. Bo S, Valpreda S, Menato G, Bardelli C, Botto C, et al. (20007) Post GDM women, despite being currently free from metabolic abnormalities, showed higher values of markers of endothelial dysfunction and IMT than controls. Atherosclerosis 194: 72-79

17. World Health Organization 1999: Definition, Diagnosis and Classification of Diabetes Mellitus and Its Complications. Report of a WHO Consultation. Part
1: Diagnosis and Classification of Diabetes Mellitus. Geneva: World Health Organisation.

18. Matthews DR, Hosker JP, Rudenski AS, Naylor BA, Treacher DF, et al. (1985) Homeostasis model assessment insulin resistance and beta cell for fasting plasma glucose and insulin in man. Diabetologia 28: 412-419.

19. Steinberg HO, Cheker H, Leowy R, Johnson A, Brechtel G et al. (1996) Obesity/ insulin resistance is associated with endothelial dysfunction: implications for the syndrome of insulin resistance. J Clin Invest 97: 2601-2610.

20. Abe Y, El-Masri B, Kimball KT, Pownell H, Reilly CF, et al. (1998) Soluble cell adhesion molecules in hypertriglyceridemia and potential significance on monocyte adhesion. Arterioscler Thrombosis and Vasc Biol 18: 723-731.

21. Bluher M, Unger R, Rossoul F, Richter V, Paschke R (2002) Relation between glycaemic control, hyperglycaemia and plasma concentrations of soluble adhesion molecules in patients with impaired glucose tolerance or type 2 diabetes. Diabetologia 45: 210-216.

22. Rizzo M, Berneis K (2007) Small dense low-density-lipoproteins and the metabolic syndrome. Diabet Metab Res Rev 23: 14-20.

23. Matsumoto K, Sera Y, Nakamura H, Ueki Y, Myiake S (2002) Serum concentrations of soluble cell adhesion molecules are related to degree of hyperglycemia and insulin resistance in patients with type 2 diabetes mellitus. Diabetes Res Clin Pract 55: 131-138.

24. Kijos SL, Buchanan TA (1991) Gestational diabetes mellitus New Engl J Med 341: 1749-1756.

25. Serne EH, RG IJ, Gans RO, Nijveldt R, De Vries G, et al. (2002) Direct evidence for insulin-induced capillary recruitment in skin of healthy subjects during physiological hyperinsulinemia. Diabetes 51: 1515-1522.

26. Sattar N, Mc Connachie A, Ford J, Gaw A, Cleland SJ, et al. (2007) Seria metabolic measurements and conversion to type 2 diabetes in the west of scotland coronary preventive study specific elevations in alanine aminotransferase and triglycerides suggest hepatic fat accumulation as contributing factor. Diabetes 56: 984-991.

27. Paradisi G, Biaggi A, Ferrazzani S, de Carolis S, Caruso A (2002) Abnorma carbohydrate metabolism during pregnancy. Association with endothelia dysfunction. Diabetes Care 25: 560-564.

28. Hu J, Norman M, Wallensteen M, Gennser G (1998) Increased large arteria stiffness and impaired acetylocholine induces skin vasodilatation in women with previous gestational diabetes mellitus. Br J Obstet Gynaecol 105: 1279-1287.

29. Wiznitzer A, Mayer A, Novack V, Sheiver E, Gilutz H, et al. (2009) Association of lipid levels during gestational diabetes with preeclampsia and gestational diabetes mellitus a population-based study. Am J Obstet Gynecol 201: 482485.

30. Sattar N (2004) Do pregnancy complications and CVD share common antecedents? Atherosclerosis 5: 3-7. 